\title{
Analysis of the non Isothermal Distributed Activation Energy Model for Biomass Pyrolysis by Fuzzy Gaussian Distribution
}

\author{
*Alok Dhaundiyal', Suraj B. Singh ${ }^{2}$ \\ ${ }^{1}$ Himgiri Zee University, Dehradun, India \\ ${ }^{2}$ G. B. Pant University of Agriculture and Technology, Pantnagar, India
}

\begin{abstract}
The main aim of this paper is to fuzzify the kinetic parameters, which have crisp nature, in order to obtain the realistic and accurate results. In the present study, the variance, upper limit of ' $\mathrm{d} E$ ' and the frequency factor are assumed to be fuzzy numbers. The Gaussian distribution is considered as the distribution function, $f$ $(E)$, of Distributed Activation Energy Model (DAEM). The membership and the non-membership functions are evaluated by the trapezoidal fuzzy number. Thermo-analytical data has been found experimentally with the help of TGA/DTG analysis. The approximated solution of DAEM is obtained with the help of asymptotic expansion.

Key words: Fuzzy number, Gaussian distribution, distributed activation energy model (DAEM), kinetic parameters, biomass pyrolysis, asymptotic expansion.
\end{abstract}

\section{Introduction}

Many engineering systems are controlled or simply analyzed through incorporating some level of imprecision about the assigned value to some parameters. When dealing with a mathematical model, one has to pay special attention towards imprecision in data. The distribution function - allows us to measure probability of parameters which directly or indirectly affect the output of a model. But the data and information gathered from the conventional sources are sometimes misleading and imprecise. Due to imprecision of the data, it sometimes becomes very complicated to evaluate the precise value of the operating parameters. For the same, fuzzy set theory was propounded by Zadeh L.A. in 1965 (Zadeh, 1965). Since it has been applied in various branches of engineering, the real question is whether replacing stubbornly imprecise data by fixed ones will influence our investigation or not. There are various pieces of evidence (Dubois \& Prade, 1980; Zadeh,1978; Kerf \& De,1975) which tell us not to count upon the Hammer principle (When you only have a hammer, you want everything at your hand to be a nail). If you substitute imprecise data by the fixed values in a model, you will leave no way around to the model but sometimes to retrieve meaningless outcomes. Fuzzification of the parameters has been found to be very efficient to curtail the imprecision associated with the data gathering. The concept of fuzzy system is much better of the classical concept, as it eliminates mathematical model of ignorance. The realistic approach involves randomness and fuzziness of operating parameters of any system.

The main aim of this paper is to fuzzify the kinetic parameters, which have crisp nature, in order to obtain the realistic and accurate results. In the present study, the variance, upper limit of ' $\mathrm{d} E$ ' and the frequency factor are assumed to be fuzzy numbers. The benefit of this approach is to shift paradigm of idealistic state of DAEM to the reality.

\section{Theory \\ Gaussian distribution function}

Gaussian distribution function is also known as "bell shaped curve" or normal distribution. Unlike binomial and Poisson distributions, the Gaussian is a continuous distribution. Probability of any unknown variable, suppose $y$, in the given interval $[a, b]$ is given by Gaussian distribution as

$$
P(a \leq y \leq b)=\frac{1}{\sigma \sqrt{2 \pi}} \int_{a}^{b} e^{-\left(\frac{(y-\mu)^{2}}{2 \sigma^{2}}\right)}
$$

where

$\mu=$ the mean of distribution,

$\sigma^{2}=$ the variance of distribution.

\footnotetext{
* Corresponding Author's email: 


\section{Distributed activation energy model}

The Distributed Activation Energy model (DAEM) or Multiple Reaction Model (MRM) is implemented to the total amount of volatile released, or to the amount of an individual volatile component like carbon monoxide or tar (Howard, 1981). It is also known as the Distributed Rate Model. It involves Vand's treatement of independent parallel processes (Pitt, 1962). Primarily, the coal devoaltization was considered first to develop the Distributed Activation
Energy Model (Vand, 1943). It involves the evaluations of double integrals, which vary rapidly and hence offer significant numerical difficulties. In order to handle the integral complication, asymptotic expansion has been used to give the solution of DAEM. Basically, the DAEM belongs to multi-reaction models. It postulates that many decomposition reactions take place, which simply includes the summation of an unlimited number of parallel single step decomposition reaction, where each reaction follows:

$$
\frac{d v_{i}}{d t}=k_{0 i} \exp \left(-\frac{E_{i}}{R T}\right)\left(v^{*}{ }_{i}-v_{i}\right)
$$

where subscript $i$ means one of several constituents, $v_{i}$ represents the total released mass of $i$ constituent, $t$ is time, $k_{\mathrm{io}}$ is the frequency factor, $E_{\mathrm{i}}$ is the activation energy, $R$ is the gas constant and $T$ is the absolute temperature.
If the number of decomposition reactions is supposed to be high enough, it can be assumed that activation energies of these reactions are distributed and the reactions can be expressed as a function of activation energy (Cai \& Ji, 2007).

$$
d v^{*}=v^{*} f(E) d E
$$

The right- hand side of equation (2) expresses the fraction of maximum mass loss $\mathrm{v}^{*}$ in the given interval of activation energy. Distribution of activation energy is assumed to follow the Gaussian distribution.
DAEM equation valid for nonisothermal time dependent temperature history, which can be derived by combination of equations (1) and (2) and it is given as:

$$
1-v=\frac{1}{\sigma \sqrt{2 \pi}} \int_{0}^{\infty} e^{\left[\frac{-k_{0}}{\beta} \int_{T_{0}}^{T} e^{\left(-\frac{E}{R T}\right)} d T-\left(\frac{\left(E-E_{0}\right)^{2}}{2 \sigma^{2}}\right)\right]} d E
$$

where $\beta$ is the heating rate and $E_{0}$ is the mean activation energy. Constant value of frequency factor $(k)$ for

\section{Asymptotic expansion}

As previously discussed, one of the main complications with the solution of the DAEM equation (3) is the evaluation of the double integral, which requires lengthy computing resources. In the previous approximations to solution of equation (3), especially in conversion of DAEM back into an equivalent single first order reaction (SFOR), lack of extrapolating to other heating regimes and difficulties in obtaining the volatile distribution $f(E)$ from the thermoanalytical data were observed. Niksa and Lau proposed a relationship between SFOR and DAEM (Niksa \& Lau,1993). every decomposition reaction with various activation energies is assumed (Cai \& Liu, 2007).

They also presented an analytical approximation to the DAEM for the exponentially and the linearly varying profiles of temperature. They have adopted an approach whose basis lies on exploiting the rapid changes occurring in the double exponential term in the equation (3).

The integrand in equation (3) contains two terms. The first term is double exponential term (DExp) dependent on time through the temperature history experienced by the sample, whereas the second part is solely dependent on the distribution of volatiles in the sample. Approximations to the double exponential term are represented as

$$
D E x p=\exp \left(-k_{0} \int_{0}^{t} e^{-\frac{E}{R T}} d t\right)
$$


In order to carry out the stepwise simplification of the integrand in the equation (4), it is very essential to assume typical values of the parameters and functions on which it depends.
The range of frequency factor $\left(k_{0}\right)$ varies from $10^{10}$ $-10^{13} \mathrm{~s}^{-1 ;}$ while the activation energies, in the range of $100-300 \mathrm{~kJ} \mathrm{~mol}^{-1}$. The temperature profile is assumed to vary linearly with time $(t)$ as

$$
\begin{gathered}
\mathrm{T}=\mathrm{mt}, \mathrm{DExp} \text { becomes } \\
D \operatorname{Exp}=\exp \left(-\int_{0}^{t} k_{0} e^{-\frac{E}{R m u}} d u\right) .
\end{gathered}
$$

where $m\left({ }^{\circ} \mathrm{C} \mathrm{min}^{-1}\right)$ is heating rate.

The integral in the exponent can be approximated with the help of Laplace method, where the parameter $\frac{E}{R m t}$ is assumed to be large, so the major contribution from the integral is when $u$ is near $t$, or the temperature is near its maximum. In this manner the well- known asymptotic approximation to DExp function can be expressed as:

$$
D E x p=\exp \left(-\int_{0}^{t} k_{0} e^{-\frac{E}{R m u}} d u\right) \sim \exp \left(\frac{-k_{0} R m t^{2}}{E}\right) \text { as } \frac{E}{R m t} \rightarrow \infty
$$

Approximation of equation (5) can also be expressed in the form

$$
D \exp =\exp \left(-\exp \left(\frac{E_{s}-E}{E_{w}}\right)\right),
$$

where the function varies from zero to one as $E$ increases over a range of step size $E_{w}$ around the central value $E_{s}$.

$$
\begin{gathered}
\text { Let } \square h(E)=\frac{E_{S}-E}{E_{w}} \text {, then equation (5) can be written as } \\
D \exp =\exp (-\exp (h(E))), \\
\text { where } \\
h(E)=\frac{-E}{R m t}+\ln \left(\frac{k_{0} R m t^{2}}{E}\right) .
\end{gathered}
$$

The behaviour of $E_{\mathrm{s}}$ is of interest, so $h(x)$ is, expanded by using Taylor series

$$
h(E) \sim h\left(E_{S}\right)+\left(E-E_{S}\right) h^{\prime}\left(E_{S}\right)+\cdots
$$

Now $h(E), E_{s}$ and $E_{w}$ are chosen in such a manner, so that

$$
h\left(E_{S}\right)=0 \text { and } h^{\prime}\left(E_{S}\right)=\frac{-1}{E_{w}} .
$$

After solving, we have

$$
E_{s}=R m t Y\left(k_{0} t\right) \text { and } E_{w}=\frac{R m t E_{s}}{R m t+E_{S}}
$$

where $Y(x)$ represents LambertW function and defined to be one of roots of the equation. 
As it has been observed that DExp varies like a smoothed step-function and rises rapidly for the large values of $t k_{0}$ from zero to one in a range of activation energies of step-size width $E_{w}$ around the value $E=E_{s}$, where both $E_{s}$ and $E_{w}$ are function of time.
In order to demonstrate the approach, consider the special case where the initial distribution $f(E)$ is Gaussian, centered at $E_{0}$ with standard deviation $\sigma$. Approximations are sought to the integral

$$
\begin{gathered}
v=\int_{0}^{\infty} \exp (h(E)) d E, \\
\text { where } \\
h(E)=-\exp \left(\frac{E_{S}-E}{E_{w}}\right)-\frac{\left(E-E_{0}\right)^{2}}{2 \sigma^{2}}
\end{gathered}
$$

and $E_{\mathrm{s}}$ and $E_{\mathrm{w}}$ are the functions of time $(t)$.

Energy is now rescaled as $y=\frac{E_{S}}{E_{0}}$, so that problem becomes

$$
\begin{gathered}
v=\sqrt{\frac{\beta}{\pi}} \int_{0}^{\infty} \exp \left[-\exp \left(\frac{y_{s}-y}{y_{w}}\right)-\beta(y-1)^{2}\right] d y \\
h(y)=-\exp \left(\frac{y_{s}-y}{y_{w}}\right)-\beta(y-1)^{2}
\end{gathered}
$$

where the constant parameter $\beta=\frac{E_{0}{ }^{2}}{2 \sigma^{2}}$. Note that in practice $1 \ll \beta$.

The concept of asymptotic expansion was presented by McGuinness M.J. et al. (McGuinness et al., 1999), which is found to provide accurate approximation for relatively narrow distribution $f(E)$, particularly for ramping temperature profile satisfying $\sigma<\sqrt{2} R T_{\mathrm{c}}$, where $T_{\mathrm{c}}$ is the temperature at which inflexion occurs in plot of released volatiles versus temperature. The method of approximation that has been discussed in this paper is similar to narrow distribution, but the approach included the simultaneous change in the behav- iour of the initial distribution $f(E)$. The term DExp is analogous to the method of the moving maximum for Laplace problems (Bender \& Orszag, 1978).

Suppose that $\sigma<R T$ for the linear variation of temperature with time. The limit adopted to demonstrate the narrow distribution is $1 \ll \sigma y_{w}$. The value of $y_{e}$ of $y$ is evaluated, where the function $h(y)$ is given in equation (8) and it is at an extremum by finding the point where $h(y)=0$. Hence

$$
h\left(y_{e}\right)^{\prime}=\frac{1}{y_{w}} \exp \left(\frac{y_{s}-y}{y_{w}}\right)-2 \beta\left(y_{e}-1\right)
$$

It is necessary to find $y_{e}$ that satisfies the equation

$$
\exp \left(\frac{y_{s}-y_{e}}{y_{w}}\right)=2 \beta y_{w}\left(y_{e}-1\right)
$$


Finally, the solution is given by

$$
y_{e}=1+y_{w} Y\left(\frac{1}{2 \beta y_{w}^{2}} \exp \left(\frac{y_{s}-1}{y_{w}}\right)\right)
$$

A Taylor series expansion $g(y)$ about $y_{e}$, truncated after quadratic terms, gives

$$
h(y) \sim h\left(y_{e}\right)+\frac{\left(y-y_{e}\right)^{2} h^{\prime \prime}\left(y_{e}\right)}{2}+\cdots
$$

The second derivative of $h^{\prime \prime}(y)$ is obtained as

$$
h\left(y_{e}\right)^{\prime \prime}=-\frac{1}{y_{w}^{2}} \exp \left(\frac{y_{s}-y_{e}}{y_{w}}\right)-2 \beta
$$

The leading behaviour of $v$ in equation (11) is given by

$$
v \sim \frac{\exp \left(h\left(y_{e}\right)\right)}{\sqrt{\left|h^{\prime \prime}\left(y_{e}\right)\right|}}
$$

This may also be written as

$$
v \sim \frac{\exp \left[-\beta\left(y_{e}-1\right)\left(2 y_{w}+y_{e}-1\right)\right]}{\sqrt{\left(1+\frac{y_{e}-1}{y_{w}}\right)}}
$$

\section{Fuzzy set}

The concept of fuzzy set theory was introduced by Zadeh L.A. (Zadeh, 1965), which is based on the assumption that the membership degree is equal to one minus non membership degree. In real life situation an object may or may not be a member of the set A to a certain degree. In other words, some hesitation about the degree of belongingness may exist. The idea of fuzzy set is in tune with human representation of reality where are more nuances than clear cut. Some philo- sophically related issues ranging from ontological level to application level via epistemological level may be found elsewhere (Zadeh, 1978).

In a fuzzy set, the membership degree of an element is expressed by any real number from 0 to 1 rather than the limiting extremes. More formally, a fuzzy set of a set $A \neq \phi$ is characterized by a membership function

$$
\xi: A \rightarrow[0,1]
$$

\section{Membership and non- membership function}

Now we will consider that the activation energy, frequency factor and variance are not clearly defined

and are fuzzy, so we will replace them with fuzzy numbers as

$$
\begin{aligned}
& \widetilde{E}=\left\{a_{1} a_{2} a_{3} a_{4} a_{1}^{\prime} a_{2} a_{3} a_{4}^{\prime}\right\} \\
& \widetilde{k_{0}}=\left\{b_{1} b_{2} b_{3} b_{4} b_{1}^{\prime} b_{2} b_{3} b_{4}{ }^{\prime}\right\} \\
& \widetilde{\sigma^{2}}=\left\{c_{1} c_{2} c_{3} c_{4} c_{1}{ }^{\prime} c_{2} c_{3} c_{4}{ }^{\prime}\right\}
\end{aligned}
$$


and define its membership $\xi_{\widetilde{E}}, \xi_{\widetilde{k_{0}}}, \xi_{\widetilde{\sigma}}$ and non-membership $\lambda_{\widetilde{E}}, \lambda_{{\widetilde{k_{0}}}_{0}}, \lambda_{\widetilde{\sigma}}$ in the following manner:

$$
\begin{aligned}
& \xi_{\tilde{E}}=\left\{\begin{array}{cl}
\frac{E-a_{1}}{a_{2}-a_{1}}, & a_{1} \leq E \leq a_{2} \\
1, & a_{2} \leq E \leq a_{3} \\
\frac{a_{4}-E}{a_{4}-a_{3}}, & a_{3} \leq E \leq a_{4} \\
0, & \text { otherwise }
\end{array}\right. \\
& \xi_{\widetilde{k}_{0}}=\left\{\begin{array}{cc}
\frac{k_{0}-b_{1}}{b_{2}-b_{1}}, & b_{1} \leq k_{0} \leq b_{2} \\
1, & b_{2} \leq k_{0} \leq b_{3} \\
\frac{b_{4}-k_{0}}{b_{4}-b_{3}}, & b_{3} \leq k_{0} \leq b_{4} \\
0, & \text { otherwise }
\end{array}\right. \\
& \xi_{\widetilde{\sigma^{2}}}=\left\{\begin{array}{cl}
\frac{\sigma^{2}-c_{1}}{c_{2}-c_{1}}, & c_{1} \leq \sigma \leq c_{2} \\
1, & c_{2} \leq \sigma \leq c_{3} \\
\frac{c_{4}-\sigma^{2}}{c_{4}-c_{3}}, & c_{3} \leq \sigma \leq c_{4} \\
0, & \text { otherwise }
\end{array}\right. \\
& \lambda_{\widetilde{E}}=\left\{\begin{array}{cl}
\frac{E-a_{1}{ }^{\prime}}{a_{2}-a_{1}{ }^{\prime}}, & a_{1}{ }^{\prime} \leq E \leq a_{2} \\
0, & a_{2} \leq E \leq a_{3} \\
\frac{a_{4}{ }^{\prime}-E}{a_{4}{ }^{\prime}-a_{3}}, & a_{3} \leq E \leq a_{4}{ }^{\prime} \\
1, & \text { otherwise }
\end{array}\right. \\
& \lambda_{\widetilde{k_{0}}}=\left\{\begin{array}{cc}
\frac{k_{0}-b_{1}{ }^{\prime}}{b_{2}-b_{1}{ }^{\prime}}, & b_{1}{ }^{\prime} \leq k_{0} \leq b_{2} \\
0, & b_{2} \leq k_{0} \leq b_{3} \\
\frac{b_{4}{ }^{\prime}-k_{0}}{b_{4}{ }^{\prime}-b_{3}}, & b_{3} \leq k_{0} \leq b_{4}{ }^{\prime} \\
1, & \text { otherwise }
\end{array}\right. \\
& \lambda_{\widetilde{\sigma^{2}}}=\left\{\begin{array}{cl}
\frac{\sigma^{2}-c_{1}{ }^{\prime}}{c_{2}-c_{1}}, & c_{1}{ }^{\prime} \leq \sigma \leq c_{2} \\
0, & c_{2} \leq \sigma \leq c_{3} \\
\frac{c_{4}{ }^{\prime}-\sigma^{2}}{c_{4}{ }^{\prime}-c_{3}}, & c_{3} \leq \sigma \leq c_{4}{ }^{\prime} \\
1, & \text { otherwise }
\end{array}\right.
\end{aligned}
$$


The $\alpha$-cut of above functions is obtained as follows:

$$
\begin{aligned}
& \widetilde{E}[\alpha]=\left\{\left[a_{1}+\alpha\left(a_{2}-a_{1}\right), a_{4}-\alpha\left(a_{4}-a_{3}\right)\right]\left[a_{1}{ }^{\prime}+\alpha\left(a_{2}-a_{1}\right), a_{4}{ }^{\prime}-\alpha\left(a_{4}{ }^{\prime}-a_{3}\right)\right]\right\} \\
& \widetilde{k_{0}}[\alpha]=\left\{\left[b_{1}+\alpha\left(b_{2}-b_{1}\right), b_{4}-\alpha\left(b_{4}-b_{3}\right)\right]\left[b_{1}{ }^{\prime}+\alpha\left(b_{2}-b_{1}{ }^{\prime}\right), b_{4}{ }^{\prime}-\alpha\left(b_{4}{ }^{\prime}-b_{3}\right)\right]\right\} \\
& \widetilde{\sigma^{2}}[\alpha]=\left\{\left[c_{1}+\alpha\left(c_{2}-c_{1}\right), c_{4}-\alpha\left(c_{4}-c_{3}\right)\right]\left[c_{1}{ }^{\prime}+\alpha\left(c_{2}-c_{1}{ }^{\prime}\right), c_{4}{ }^{\prime}-\alpha\left(c_{4}{ }^{\prime}-c_{3}\right)\right]\right\}
\end{aligned}
$$

After applying the $\alpha$-cut, the central value $E_{\mathrm{s}}$ will reduce to the form

$$
\begin{gathered}
E_{S}=\left\{[ \operatorname { R m t } Y ( \{ b _ { 1 } + \alpha ( b _ { 2 } - b _ { 1 } ) \} t ) , \operatorname { R m t } Y ( \{ b _ { 4 } - \alpha ( b _ { 4 } - b _ { 3 } ) \} t ) ] \left[\operatorname { R m t } Y \left(\left\{b_{1}{ }^{\prime}\right.\right.\right.\right. \\
\left.\left.\left.\left.+\alpha\left(b_{2}-b_{1}\right)\right\} t\right), \operatorname{Rmt} Y\left(\left\{b_{4}{ }^{\prime}-\alpha\left(b_{4}{ }^{\prime}-b_{3}\right)\right\} t\right)\right]\right\}
\end{gathered}
$$

\section{Fuzzy Gaussian distribution function}

If the remaining mass fraction of biomass sample is modeled by the Gaussian distribution, then

$$
f(E)=e^{\left[\frac{-k_{0}}{\beta} \int_{T_{0}}^{T} e^{\left(-\frac{E}{R T}\right)} d T-\left(\frac{\left(E-E_{0}\right)^{2}}{2 \sigma^{2}}\right)\right]}, E>0, k_{0}>0, \sigma>0
$$

where $\mathrm{E}$ (activation energy), $k_{0}$ ( frequency factor), ability of obtaining a value in the interval $[a, b]$, is as and $\sigma$ (variance) are crisp in nature. Let fuzzy numbers $\quad \tilde{P}(a \leq X \leq b)$ and compute its $\alpha$-cut as follows.

$\widetilde{E}, \widetilde{k_{0}}, \tilde{\sigma}$ replace $E, E_{k}$ and $\sigma$. Then, the fuzzy prob-

$$
\begin{aligned}
& \left.\tilde{P}(a<v<b)[\alpha]=\left\{\int_{a}^{b} e^{\left[\frac{-\bar{k}_{0}}{\beta} \int_{T_{0}}^{T} e^{\left(-\frac{\widetilde{E}}{R T}\right)} d T-\left(\frac{\left(\widetilde{E}-E_{0}\right)^{2}}{2 \widetilde{\sigma}^{2}}\right)\right.}\right] d E \mid E \in \tilde{E}[\alpha]\right\}=\left[P^{L}[\alpha], P^{U}[\alpha]\right], \\
& \left.\tilde{P}(a<v<b)[\alpha]=\left\{\int_{a}^{b} e^{\left[\frac{-\widetilde{k_{0}}}{\beta} \int_{T_{0}}^{T} e^{\left(-\frac{\widetilde{E}}{R T}\right)} d T-\left(\frac{\left(\widetilde{E}-E_{0}\right)^{2}}{2 \widetilde{\sigma}^{2}}\right)\right.}\right] d E \mid k_{0} \in \widetilde{k_{0}}[\alpha]\right\}=\left[P^{L}[\alpha], P^{U}[\alpha]\right], \\
& \left.\tilde{P}(a<v<b)[\alpha]=\left\{\int_{a}^{b} e^{\left[\frac{-k_{0}}{\beta} \int_{T_{0}}^{T} e^{\left(-\frac{\widetilde{E}}{R T}\right)} d T-\left(\frac{\left(\widetilde{E}-E_{0}\right)^{2}}{2 \widetilde{\sigma}^{2}}\right)\right.}\right] d E \mid \sigma \in \tilde{\sigma}[\alpha]\right\}=\left[P^{L}[\alpha], P^{U}[\alpha]\right],
\end{aligned}
$$

for all $\alpha$, where

$$
\begin{aligned}
& P^{L}=\min \left\{e^{\left[\frac{\widetilde{-k_{0}}}{\beta} \int_{T_{0}}^{T} e^{\left(-\frac{\widetilde{E}}{R T}\right)} d T-\left(\frac{\left(\widetilde{E}-E_{0}\right)^{2}}{2 \widetilde{\sigma}^{2}}\right)\right]} \mid E \in \tilde{E}[\alpha]\right\} \\
& P^{U}=\max \left\{e^{\left[\frac{-\bar{k}_{0}}{\beta} \int_{T_{0}}^{T} e^{\left(-\frac{\widetilde{E}}{R T}\right)} d T-\left(\frac{\left(\widetilde{E}-E_{0}\right)^{2}}{2 \widetilde{\sigma}^{2}}\right)\right]} \mid E \in \tilde{E}[\alpha]\right\}
\end{aligned}
$$




$$
\begin{aligned}
& P^{L}=\min \left\{e^{\left[\frac{\widetilde{-k_{0}}}{\beta} \int_{T_{0}}^{T} e^{\left(-\frac{\widetilde{E}}{R T}\right)} d T-\left(\frac{\left(\widetilde{E}-E_{0}\right)^{2}}{2 \widetilde{\sigma}^{2}}\right)\right]} \mid k_{0} \in \widetilde{k_{0}}[\alpha]\right\} \\
& P^{U}=\max \left\{e^{\left[\frac{\widetilde{-k_{0}}}{\beta} \int_{T_{0}}^{T} e^{\left(-\frac{\widetilde{E}}{R T}\right)} d T-\left(\frac{\left(\widetilde{E}-E_{0}\right)^{2}}{2 \widetilde{\sigma}^{2}}\right)\right]} \mid k_{0} \in \widetilde{k_{0}}[\alpha]\right\} \\
& P^{L}=\min \left\{\mathrm{e}^{\left[\frac{-\mathrm{-k}_{0}}{\beta} \int_{\mathrm{T}_{0}}^{\mathrm{T}} \mathrm{e}^{\left(-\frac{\widetilde{\mathrm{E}}}{\mathrm{RT}}\right)} \mathrm{dT}-\left(\frac{\left(\widetilde{\mathrm{E}}-\mathrm{E}_{0}\right)^{2}}{2 \tilde{\sigma}^{2}}\right)\right]} \mid \sigma \in \tilde{\sigma}[\alpha]\right\} \\
& \mathrm{P}^{\mathrm{U}}=\max \left\{\mathrm{e}^{\left[\frac{-\mathrm{-k}_{0}}{\beta} \int_{\mathrm{T}_{0}}^{\mathrm{T}} \mathrm{e}^{\left(-\frac{\widetilde{\mathrm{E}}}{\mathrm{RT}}\right)} \mathrm{dT}-\left(\frac{\left(\widetilde{\mathrm{E}}-\mathrm{E}_{0}\right)^{2}}{2 \widetilde{\sigma}^{2}}\right)\right]} \mid \sigma \in \tilde{\sigma}[\alpha]\right\}
\end{aligned}
$$

\section{Application}

For applications, the experiment has been conducted for the non-isothermal pyrolysis of pine needles. It is to be noted that the result of this paper has been used in the fuzzification process to obtain the precise and authentic value of parameters with the help of DAEM.
Table1 indicates the chemical composition which is also obtained with the help of CHNO-S (carbon, hydrogen, nitrogen, oxygen, and sulphur) analyser of pine needle samples.

\section{Chemical composition of pine needles}

\begin{tabular}{|l|l|l|l|l|l|l|l|l|}
\hline Biomass Type & C & H & N & O & V.M $^{*}$ & H.H.V & S & Ash \\
\hline Pine Needle & 53.64 & 5.36 & 0.62 & 33.92 & 68.4 & 20.8 & 0.20 & 2.1 \\
\hline
\end{tabular}

*Volatile matter **High heating value

\section{Numeral illustration}

Assuming the initial distribution of activation energy follows the Gaussian distribution with fuzzy scale parameters $\widetilde{E}, \widetilde{k_{0}}$ and $\tilde{\sigma}$ taken as the trapezoidal fuzzy number.
The values of parameters obtained after fuzzification are taken at $\alpha=0$.

Let $\tilde{E}\left(\mathrm{kJmol}^{-1}\right)=[(14386,21352,28318,42250)], \widetilde{k_{0}}\left(s^{-1}\right)=$ $[(3.96,4.92,34.56,408)]$ and $\widetilde{\sigma^{2}}\left(\mathrm{kJmol}^{-1}\right)=[(1.44 e-06,0.09486,0.64,9.010)]$.

The effect of fuzzy parameters with temperature is represented by means of fuzzified bands $R e_{1}, R e_{2}$, $R e_{3}, R e_{4}$. The $R e_{1}$ and $R e_{2}$ corresponding to membership function; whereas $R_{3}$ and $R e_{4}$ represents those corresponding to non-membership function. After the fuzzification of outer limit of " $\mathrm{d} E$ " integral, $\widetilde{E}$, we have obtained four fuzzified bands which are depicted in
Figure 1. If the nature of the outer limit of " $\mathrm{d} E$ " integral, $E$, had been crisp in nature, all the four fuzzified bands would have been converged to a single band. At the beginning of pyrolysis reaction, the remaining mass proportion must be close to one. From the fuzzified bands obtained in Figure 1, it has been seen that the remaining mass fraction agrees to thermo analytical data. 


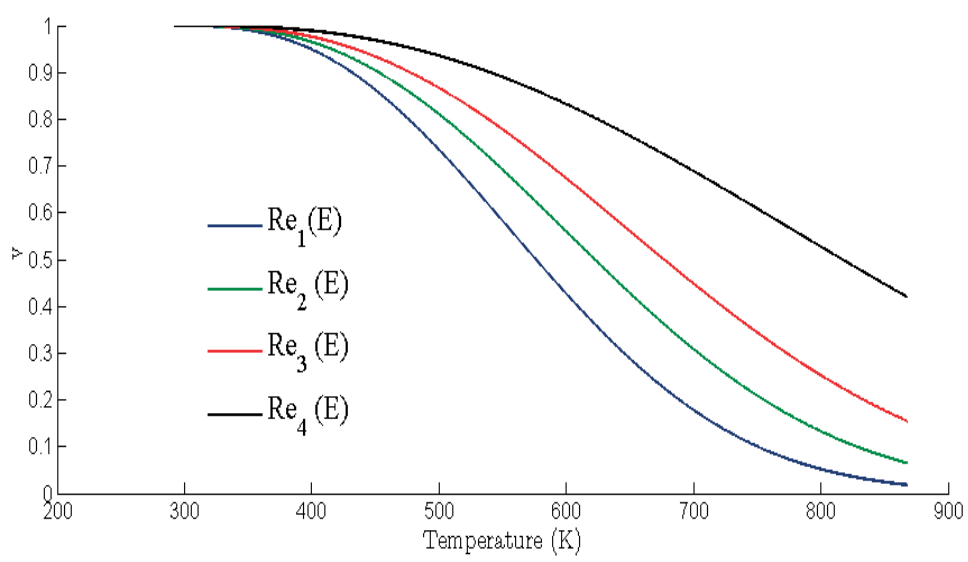

Figure 1: The effect of fuzzified upper limit $(\widetilde{E}$,$) of “ \mathrm{d} E$ " integral on the numerical results.

The effect of the fuzzified frequency factor on the numerical results is shown in Figure 2.

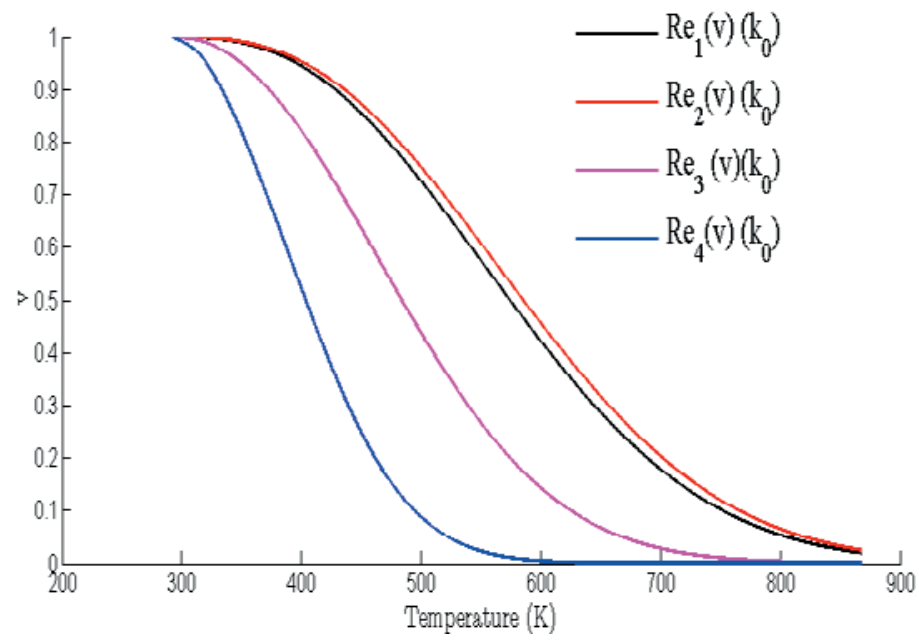

Figure 2: The effect of fuzzified frequency factor $\left(\widetilde{k_{0}}\right)$ on the numerical solution of DAEM.

According to the fuzzified bands, an increase in width of fuzzy subset causes fuzzified bands leading to left direction. The effect of fuzzification of variance is illustrated in Figure 3, where it is observed that remaining mass fraction curves approach to one with a decrease in the size of interval of membership function.
Cai and Liu (Cai \& Liu, 2007) used the crisp logic to describe the influence of relevant parameters on biomass proylosis. Comparatively, the fuzzy logic extends the possibilities of solution which is converged to a single band by Cai and Liu (Cai \& Liu, 2007). 


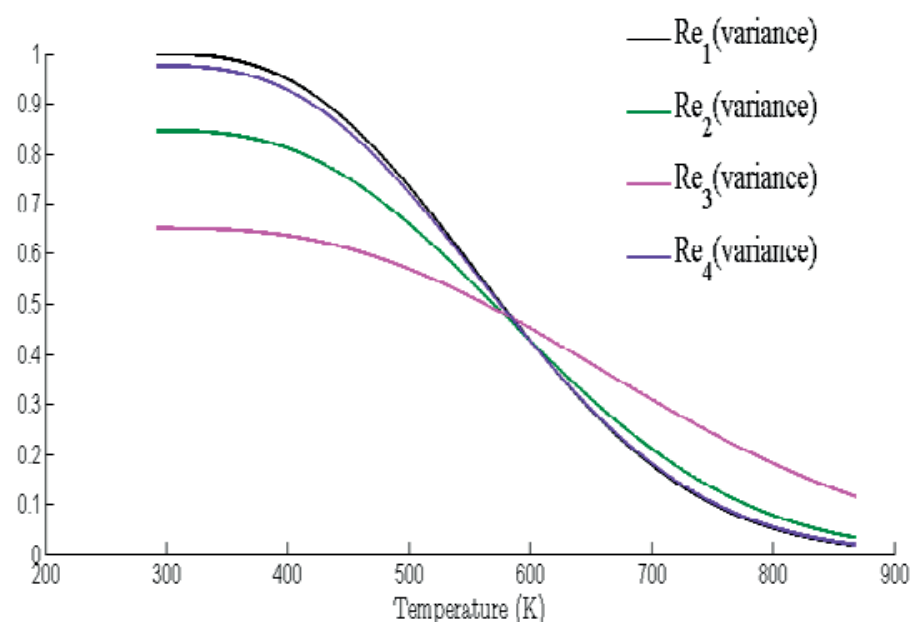

Figure 3: The effect of fuzzified variance $(\tilde{\sigma})$ of Gaussian distribution on the numerical solution of DAEM.

\section{Conclusions}

In this paper the kinetic parameters of DAEM have been taken in fuzzy form. The fuzzy system has been successfully applied to the DAEM. Whenever the distribution of activation energies and the parameters of distribution function contain randomness and fuzziness respectively, conventional system is not feasible. Thus, we invoked successfully the fuzzy theory to overcome this complication. The use of fuzzified- kinetic parameters in the distributed activation energy model has overshadowed the demerits of the crisp-kinetic parameters. The fuzzy sets have handled the randomness or fuzziness to a certain desired level of accuracy, which in turn helped in making our analysis more precise and realistic. The membership and the non-membership function of fuzzy set have been computed. Using this method, the fuzziness of kinetic parameters has been evaluated. The value of the fuzzified kinetic parameters is maximum in the small interval of membership function.

\section{References}

1. Bender, C.M., and Orszag, S.A. (1978). Advanced Mathematical Methods for Scientists and Engineers, McGraw-Hill, 247-316.

2. Cai, J., Ji, L.(2007). Pattern Search Method For Determination Of DAEM Kinetic Parameters From Nonisothermal TGA Data Of Biomass, Journal of Mathematical Chemistry, 42(3): 547553.

3. Cai J.M., Liu, R.H. (2007). Parametric study of the nonisothermal nth-order Distributed activation energy model involved the Weibull distribution for biomass pyrolysis, 89, 971-975.
4. Cai, J., Liu R. (2008). New distributed activation energy model: Numerical solution and application to pyrolysis kinetics of some types of biomass", Bioresource Technology, 99, 2795-2799.

5. Dubois, D. and Prade, H. (1980). Fuzzy Sets and Systems: Theory and Applications. Academic Press, 227-256.

6. Howard, J.B. (1981). Fundamentals of coal pyrolysis and hydropyrolysis, in Chemistry of Coal Utilization, Chapter 12 (Edited by M.A. Elliott), Wiley and Sons, 359-386.

7. Kerf, J.De . (1975). A bibliography on fuzzy sets. J. comput. Appl. Math 1, 205-212.

8. McGuinness, M.J., Donskoi, E., and McElwain, D.L.S. (1999). Asymptotic Approximations to the Distributed Activation Energy Model Appl. Math. Lett., 12:27-34.

9. Niksa, S., and Lau, C-W. (1993). Global rates of devolatilization for various coal types Combust. Flame 94:293.

10. Pitt, G.J. (1962). The kinetics of the evolution of volatile products from coal. Fuel 1:267.

11. Türksen, I.B. (2003). A perspective on the Philosophical Grounding of fuzzy Theories, Proceedings of IFSA, 1-15.

12. Vand, A. (1943). Theory of the irreversible electrical resistance changes of metallic films evaporated in vacuum, Proc. Phys. Soc. Lond. A (55):222.

13. Zadeh L.A. (1965). Fuzzy sets. Information and Control. 8: 338 -353.

14. Zadeh, L.A. (1978). Fuzzy sets as a basis for a theory of possibility. Fuzzy sets and systems, 1: 3-28. 